\title{
Equações Alométricas para Estimativa de Fitomassa em Cultivo de Pinhão Manso
}

\author{
Willian José Ferreira ${ }^{1}$, Getúlio Teixeira Batista ${ }^{2}$, Marcelo dos Santos Targa ${ }^{2}$, \\ Cristina Maria de Castro³, Antônio Carlos Pries Devide ${ }^{3}$, Lia Braz ${ }^{1}$ \\ ${ }^{1}$ Centro de Ciência do Sistema Terrestre - CCST, Instituto Nacional de Pesquisas Espaciais - INPE,
} São José dos Campos/SP, Brasil

${ }^{2}$ Programa de Pós Graduação em Ciências Ambientais, Universidade de Taubaté - UNITAU, Taubaté/SP, Brasil ${ }^{3}$ Setor de Fitotecnia, Agência Paulista de Tecnologia dos Agronegócios - APTA, Pindamonhangaba/SP, Brasil

\begin{abstract}
RESUMO
Este trabalho descreve estimativas de fitomassa e de estoque de carbono em cultivo de pinhão no Vale do Paraíba Paulista utilizando equações alométricas desenvolvidas com base em análise de regressão, que consideraram a fitomassa como uma variável dependente de medições dendrométricas de fácil obtenção na planta. As estimativas foram validadas por meio de método destrutivo direto, ponderando o corte de árvores de diferentes alturas e estruturas. Quando comparado com outros modelos, a equação linear $\mathrm{Y}=1,3\left(\mathrm{~h}^{2} \mathrm{~d}\right)$, em que $Y$ é a biomassa $(\mathrm{kg}), h$ é a altura $(\mathrm{m})$ e $d$ é a projeção da copa $(\mathrm{m})$, apresentou resultados confiáveis e erros não significativos para árvores de até $3,2 \mathrm{~m}$ de altura. Para o espaçamento de $4 \times 3 \mathrm{~m}$ foi avaliado que o estoque de matéria seca variou entre 0,5 e $6 \mathrm{Mg} \mathrm{ha}^{-1}$, enquanto o carbono armazenado na área pode chegar a $3 \mathrm{Mg} \mathrm{Cha}^{-1}$.
\end{abstract}

Palavras-chave: Jatropha curcas, bioenergia, mecanismo de desenvolvimento limpo.

\section{Allometric Equations for the Estimation of Jatropha Curcas Biomass}

\begin{abstract}
This paper describes the estimation of biomass and carbon stock in Jatropha crops in the Vale do Paraíba region, Brazil, using allometric equations developed based on regression analysis which considered the biomass as a variable dependent on easily obtainable dendrometric measurements. The biomass estimated using allometric equations was validated by the destructive direct method based on the cutting of trees of different heights and structures. When compared with other models, the linear equation $\mathrm{Y}=1.3\left(\mathrm{~h}^{2} \mathrm{~d}\right)$, where $Y$ is the biomass $(\mathrm{kg}), h$ is the height $(\mathrm{m})$, and $d$ is the crown projection $(\mathrm{m})$ showed reliable results and no significant errors to estimate trees up to $3.2 \mathrm{~m}$ tall. For the $4 \times 3 \mathrm{~m}$ plant spacing, result evaluations showed that the stock of dry matter ranged from 0.5 to $6 \mathrm{Mg} \mathrm{ha}^{-1}$, while the carbon stored in the area could reach $3 \mathrm{Mg} \mathrm{Cha}^{-1}$.
\end{abstract}

Keywords: Jatropha, bioenergy, clean development mechanism. 


\section{INTRODUÇÃO}

A possibilidade de alcançar elevado rendimento de grãos para a obtenção de óleos vegetais destinados à produção de combustíveis alternativos, sem ser uma espécie alimentar, faz do pinhão manso (Jatropha curcas L.) a planta que poderá sustentar a cadeia produtiva do biodiesel no Brasil (Laviola et al., 2009).

A recente introdução comercial do cultivar em regiões semiáridas e pastagens degradadas do país tornou promissora a obtenção de Reduções Certificadas de Emissões (RCE), créditos de carbono, previstos pelo Protocolo de Kyoto, por meio de atividades integradas a empreendimentos e projetos candidatos ao Mecanismo de Desenvolvimento Limpo (MDL) (Silva et al., 2012; Devide et al., 2010).

Reverter a degradação ambiental mediante a reabilitação de solos de baixa fertilidade é apontada como forte premissa ao melhoramento de sistemas produtivos (Urbanska et al., 2000), além de preservar as bases para o desenvolvimento sustentável (Medeiros, 2006).

O MDL tem considerável relevância no Brasil, que, desde a década passada, firma numerosos compromissos voluntários junto às Nações Unidas para reduzir a emissão de gases frente à projeção mundial de emissões (Helou et al., 2012). No entanto, as perspectivas de obtenção dos créditos por meio de sistemas promotores da baixa emissão de carbono vêm perdendo ímpeto em diversas regiões com plantios experimentais de pinhão manso, por decorrência, principalmente, do diminuto rendimento agrícola das plantas nesses sítios, o que frustra a expectativa dos produtores rurais que ansiavam por um rápido incremento na renda (Juhász et al., 2010).

Ainda assim, motivados por princípios orientados no UN-REDD Programme (2012), que em sua temática aborda a conservação e o manejo sustentável em áreas florestais e especifica valores financeiros ao carbono armazenado nessas áreas, persiste o interesse por propostas de compensação aos produtores que conseguirem reduzir o desmatamento e a degradação ambiental, evidenciando que os créditos poderiam amortizar os custos da produção e contribuir como renda extra.

Sob essa óptica, relata-se que árvores de pinhão manso com aproximadamente quatro anos de idade são capazes, potencialmente, de estocar entre $3 \mathrm{e} 4 \mathrm{MgCha}^{-1}$
(Torres et al., 2011; Reinhardt et al., 2007) e que dos 300 projetos de MDL registrados no Brasil (ano-base 2013) apenas três deles são associados à conservação e ao manejo sustentável de áreas florestais (Brasil, 2014). Há, portanto, um mercado potencial de crescimento nessa área. Ressalta-se que existe fomento para o desenvolvimento do ciclo completo de projetos de MDL no país (EAESP, 2012) (nenhum na linha florestal), mas existe possibilidade de abertura de novas frentes de atividades em um futuro próximo.

Mensurar a mitigação do efeito estufa por sistemas florestais é um tema bastante controverso, porém o desenvolvimento de equações específicas e regionais é apontado como uma importante ferramenta na estimativa da fitomassa e do estoque de carbono em povoamentos florestais (Leite \& Andrade, 2003).

Essas estimativas são facilitadas ao assumir-se que a fitomassa se constitui em um componente dependente de variáveis dendrométricas de fácil mensuração no dossel, tais como altura e projeções da copa, combinadas ou não (Ghezehei et al., 2009; Rolim et al., 2006).

Cole \& Ewel (2006) alertam para a dificuldade de se encontrar relações alométricas para árvores do gênero Jatropha, devido ao fato de que muitos dos modelos desenvolvidos ainda se baseiam no volume do dossel (Miranda et al., 2011), enquanto que o crescimento do pinhão manso se dá de forma modular em progressão geométrica, surgindo dois ramos a partir de cada inflorescência, assim sucessivamente (Dias et al., 2007). Logo, dentre os fatores limitantes, a complexa forma estrutural da planta aparece como barreira à formulação de equações que possam estimar, de modo satisfatório, sua fitomassa.

A continuidade de pesquisas que possam relacionar medidas de campo com estimativas de biomassa tem sua importância aumentada, especialmente, em áreas de difícil acesso (Mohan Kumar et al., 1998; Clough \& Scott, 1989).

Dessa forma, com a finalidade de se obterem dados que contribuam para a avaliação do potencial da planta para projetos de créditos de carbono, este trabalho objetivou, de modo prospectivo, estimar, por meio do ajuste de equações e modelos matemáticos já propostos, a fitomassa arbórea aérea e o armazenamento de carbono em árvores de pinhão manso no Vale do Paraíba do Sul, SP. 


\section{MATERIAL E MÉTODO}

\subsection{Caracterização da área de estudo}

A área de estudo localiza-se em uma propriedade rural no bairro Registro, município de Taubaté, no Vale do Paraíba Paulista ( $\left.23^{\circ} 8^{\prime} \mathrm{S}, 45^{\circ} 28^{\prime} \mathrm{W}\right)$, a $685 \mathrm{~m}$ de altitude.

A classificação de Köppen indica o clima regional como Cwa, com inverno frio e seco contrapondo-se a verões brandos e chuvosos (precipitação média anual de $1.346 \mathrm{~mm}$ ). A temperatura média anual é de $23{ }^{\circ} \mathrm{C}$, com a mínima de $6{ }^{\circ} \mathrm{C}$ e máxima de $37^{\circ} \mathrm{C}$ (Castro et al., 2006; Fisch, 1999).

O experimento foi instalado pela Agência Paulista de Tecnologia dos Agronegócios (APTA) em novembro de 2006, em uma área de 2,0 ha, em plantio direto na pastagem, em um Latossolo Vermelho-Amarelo (LVA), relevo ondulado com $14 \%$ de declividade, em covas abertas no espaçamento $4 \times 3 \mathrm{~m}$ (Figura 1 ).

A pesquisa da APTA foi iniciada em agosto de 2007 para determinar o balanço de energia na reciclagem de nutrientes e $\mathrm{o}$ aporte de nitrogênio no solo, via fixação biológica de leguminosas, em comparação à pastagem

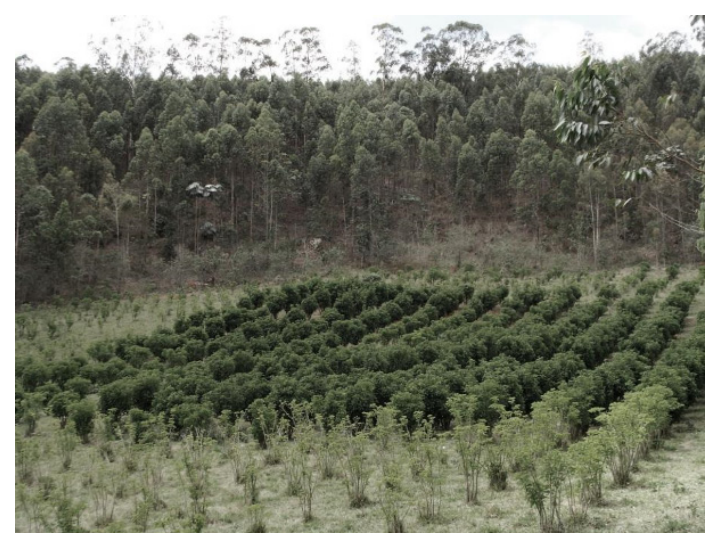

Figura 1. Área experimental cultivada com pinhão manso (primeiro plano) em Taubaté, SP.

Figure 1. Experimental area cultivated with Jatropha (foreground) in Taubaté, SP. degradada de Brachiaria brizantha (Devide et al., 2010). Dentre outros aspectos investigados, avaliaram-se também os efeitos da poda no pinhão manso cultivado junto a essas diferentes coberturas vegetais.

Inicialmente, caracterizou-se o solo e aplicou-se calcário a lanço na área, seguido da passagem de riscador a $5 \mathrm{~cm}$ de profundidade. Após essa etapa, as parcelas receberam o plantio de leguminosas herbáceas perenes (LHP) como culturas de coberturas (amendoim - Arachis pintoi e calopogônio - Calopogonium mucunoides) nas entrelinhas do pinhão manso. As parcelas não foram irrigadas e nem se aplicou trato cultural específico.

Desde a instalação do experimento, toda a área foi isolada por cerca, a fim de que o solo não fosse pisoteado por animais e, neste estudo, a avaliação da fitomassa da parte aérea das árvores foi conduzida dentro da área total de estudo, em parcelas sem poda e consorciadas com a pastagem.

Em julho de 2012, antecedendo a avaliação da fitomassa do pinhão manso, foram determinadas as características físico-químicas do solo, que apresentou textura arenoargilosa, com densidade média de $1,4 \pm 0,1 \mathrm{~g} \mathrm{~cm}^{-3} \mathrm{e}$ caracterização química apresentada na Tabela 1 .

\subsection{Determinação da massa seca do pinhão manso por método direto}

A quantificação da massa seca dos elementos da parte aérea do pinhão manso foi efetuada por meio do corte de 11 árvores, amostradas ao acaso, considerando-se diferentes alturas e diferentes classes diamétricas, dentro da área total de estudo, na parcela das árvores sem poda na área estabelecida em plantio direto junto a pastagem de braquiária, conforme método direto $\mathrm{e}$ destrutivo descrito por Watzlawick et al. (2002).

Nesse procedimento, cada árvore foi desfolhada e suas folhas foram ensacadas e pesadas para que, no laboratório, fosse determinado o peso seco das folhas em relação ao peso fresco.

Tabela 1. Caracterização química de duas camadas do solo da área experimental.

Table 1. Chemical characteristics of two soil layers of the experimental área.

\begin{tabular}{lccccccccccccc} 
& $\mathbf{p H}$ & $\mathbf{M O}$ & $\mathbf{P}$ & $\mathbf{K}$ & $\mathbf{C a}$ & $\mathbf{M g}$ & $\mathbf{H}+\mathbf{A l}$ & $\mathbf{B}$ & $\mathbf{C u}$ & $\mathbf{F e}$ & $\mathbf{M n}$ & $\mathbf{Z n}$ \\
\hline & & $g d^{-3}$ & $m g d m^{-3}$ & & \multicolumn{2}{c}{$m m o l d m^{-3}$} & & & & $m g d m^{-3}$ & & \\
Superfície & 5,4 & 32 & 30 & 1,1 & 40 & 16 & 22 & 0,2 & 1,1 & 48 & 48,3 & 5,0 \\
$\mathbf{3 0 - 4 0}(\mathbf{c m})$ & 4,6 & 10 & 4 & 0,6 & 14 & 4 & 30 & 0,2 & 0,9 & 34 & 48,1 & 1,2 \\
\hline
\end{tabular}


Ainda em campo, depois do desfolhamento, antecedendo ao corte de cada árvore, foram medidas com uma fita métrica a altura, o diâmetro e o raio de projeção da copa no solo. Em seguida, procedeu-se ao corte e, com o auxílio de um dinamômetro, efetuou-se a pesagem dos ramos. Amostras desses foram retiradas para a estimativa da umidade e determinação do peso de matéria seca, segundo o método da proporcionalidade (Soares et al., 2007). Essas amostras úmidas das folhas e dos ramos foram colocadas em estufa com circulação e renovação de ar, a uma temperatura de aproximadamente $65^{\circ} \mathrm{C}$, até a estabilização do peso de matéria seca.

\subsection{Equações para estimativa da fitomassa e armazenagem de carbono}

Com base nas medidas das 11 árvores amostradas, utilizadas no processo de determinação da massa seca, ajustaram-se os dados usando-se análise de regressão, observando-se previamente a relação entre o grupo de variáveis e a fitomassa da planta, pela definição de que somente as variáveis com correlação linear de Pearson (r) superior a 95\% deveriam ser utilizadas no modelo (Cole \& Ewel, 2006).

$\mathrm{O}$ ajuste da regressão foi realizado pelo método dos mínimos quadrados, associado à observação de aspectos relacionados à análise de variância e de resíduos e à amplitude de probabilidade entre os valores estimados e observados.
Duas árvores foram separadas dentre as amostradas para a validação da equação gerada ao final do processo, que não foram utilizadas na análise de regressão.

Após a avaliação da significância estatística, a equação foi comparada com outras três equações desenvolvidas para obtenção de estimativas da fitomassa do pinhão manso para os cultivos entre $3 \mathrm{e} 4$ anos de idade (Toledo et al., 2012; Ghezehei et al., 2009).

Por fim, o intervalo estimativo da massa seca total da área experimental foi obtido pela multiplicação dos valores máximos e mínimos pelo número de árvores de pinhão manso por hectare.

A partir desses dados foram também estimados os limites, superior e inferior, do estoque de carbono fixado na área, por meio do produto da massa seca do pinhão manso obtida em laboratório e utilizando-se o fator 0,5 (Dewar \& Cannell, 1992). Posteriormente, o armazenamento de carbono foi extrapolado para megagrama por hectare $\left(\mathrm{Mg} \mathrm{Cha}^{-1}\right)$, considerando-se o espaçamento de $4 \times 3 \mathrm{~m}$ utilizado no experimento.

\section{RESULTADOS E DISCUSSÃO}

\subsection{Determinação da fitomassa seca por método direto}

A Tabela 2 apresenta a descrição das 11 árvores amostradas e a fitomassa do pinhão manso obtida por meio da pesagem que antecedeu seu corte em campo.

Tabela 2. Estatística descritiva das árvores amostras avaliadas no experimento.

Table 2. Descriptive statistics of sampled trees evaluated in the experiment.

\begin{tabular}{|c|c|c|c|c|c|}
\hline Árvores amostradas & Altura (h) & Diâmetro $(\mathbf{d})$ * & Raio $(\mathbf{r})^{*}$ & Volume $(\mathrm{V})^{* *}$ & Fitomassa \\
\hline $\mathbf{N}$ & & $m$ & & $m^{3}$ & $k g$ \\
\hline 1 & 1,9 & 1,2 & 0,7 & 0,6 & 7,5 \\
\hline 2 & 2,2 & 2,0 & 0,9 & 1,0 & 10,0 \\
\hline 3 & 2,8 & 1,6 & 0,7 & 0,8 & 16,8 \\
\hline 4 & 3,2 & 2,2 & 0,8 & 1,1 & 29,8 \\
\hline 5 & 2,7 & 1,6 & 0,7 & 0,8 & 13,3 \\
\hline 6 & 1,5 & 0,9 & 0,7 & 0,4 & 3,7 \\
\hline 7 & 2,7 & 1,4 & 0,7 & 0,7 & 11,2 \\
\hline 8 & 2,8 & 1,6 & 0,7 & 0,8 & 15,9 \\
\hline 9 & 1,7 & 0,8 & 0,6 & 0,3 & 3,0 \\
\hline 10 & 1,1 & 0,5 & 0,7 & 0,3 & 2,0 \\
\hline 11 & 2,9 & 2,4 & 0,8 & 0,9 & 26,2 \\
\hline Mínimo & 1,1 & 0,5 & 0,6 & 0,3 & 2,0 \\
\hline Máximo & 3,2 & 2,4 & 0,9 & 1,1 & 29,8 \\
\hline Média & 2,3 & 1,5 & 0,7 & 0,7 & 12,7 \\
\hline Desvio padrão & 0,7 & 0,61 & 0,1 & 0,3 & 9,1 \\
\hline
\end{tabular}

*em relação à projeção da copa. ${ }^{* * V o l u m e ~(V) ~ c a l c u l a d o ~ a ~ p a r t i r ~ d e ~ G h e z e h e i ~ e t ~ a l . ~(2009) . ~}$ 
A distribuição percentual da massa relativa do lenho, casca e folhas não sofreu modificações significativas nas diferentes plantas e foi observado que para árvores mais altas, maiores do que $2 \mathrm{~m}$ de altura, a composição média encontrada foi $74 \%$ para o lenho, $23 \%$ para a casca e $3 \%$ para as folhas, enquanto que para árvores de menor estatura os valores para essas partes foram respectivamente $69 \%, 29 \%$ e $2 \%$.

A relação comparativa entre massa seca e fresca foi linear para todas as partes analisadas e o coeficiente de determinação, elevado $\left(r^{2}=0,93\right)$, com o peso de matéria seca, em média, correspondendo a $23 \%$ da massa úmida do pinhão manso, resultado significativo a $5 \%$ de probabilidade, pelo teste $t$ de Student.

\subsection{Ajuste de equações alométricas para estimativa de fitomassa}

As características das variáveis estudadas em relação à fitomassa medida em campo foram avaliadas por meio de uma matriz de correlação que na Tabela 3 tem os resultados da correlação linear de Pearson (r) e p-valor apresentados.

Embora outras variáveis tenham apresentado coeficiente linear alto e significativo isoladamente, $\mathrm{h}^{2} \mathrm{~d}$ apontou a correlação mais alta e significativa em relação à fitomassa do pinhão manso e p-valor não significativo a $5 \%$ de probabilidade pelo teste $t$ de Student, resultado sinalizador de que o modelo que relaciona $\mathrm{h}^{2} \mathrm{~d}$ com a fitomassa do pinhão manso de maneira simples e direta é o mais indicado.

Portanto, a análise do conjunto de dados associados às medidas de $\mathrm{h}^{2} \mathrm{~d}$ e da fitomassa, respectivamente, variável regressora e variável de resposta, resultou na equação alométrica Equação 1:

$\mathrm{Y}=1,3\left(\mathrm{~h}^{2} \mathrm{~d}\right)$

em que: $\mathrm{Y}=$ fitomassa estimada $(\mathrm{kg}) ; \mathrm{h}=$ altura da copa (m); d = diâmetro da copa (m); 1,3 - coeficiente determinado na regressão linear.

Como primeira avaliação desse modelo (Equação 1), investigou-se o comportamento dos dados das duas árvores-teste, então separadas da análise de regressão. A Figura 2 ilustra a relação encontrada entre os valores preditos por meio da Equação 1 versus os valores observados em campo.
A análise de variância da Equação 1 é apresentada na Tabela 4.

O teste de hipótese revelou que a equação foi significativa ao nível de $5 \%$ de probabilidade no teste F para a estimativa da fitomassa, porém o p-valor não foi significativo para as massas calculadas, reforçando o caráter probabilístico existente entre as variáveis.

Observada de modo gráfico na Figura 3, a distribuição da amplitude dos resíduos possibilita a observação de informações complementares sobre o ajuste do modelo.

Tabela 3. Correlação linear entre variáveis medidas e combinadas e a fitomassa do pinhão manso.

Table 3. Correlation between measured variables and biomass of Jatropha.

\begin{tabular}{ccc}
\hline Variáveis & r (Pearson) & p-valor \\
\hline $\mathrm{h}$ & 0,95 & $\mathrm{NS}$ \\
$\mathrm{d}$ & 0,92 & $>0,005$ \\
$\mathrm{~V}$ & 0,93 & $\mathrm{NS}$ \\
\hline $\mathrm{h}^{2}$ & 0,97 & $\mathrm{NS}$ \\
\hline $\mathrm{h}^{2} \mathrm{~d}$ & 0,99 & $\mathrm{NS}$ \\
\hline $\mathrm{d}^{2}$ & 0,92 & $\mathrm{NS}$ \\
\hline $\mathrm{hd}^{2}$ & 0,97 & $\mathrm{NS}$ \\
\hline $\mathrm{dh}$ & 0,97 & $\mathrm{NS}$ \\
\hline
\end{tabular}

Diferença não significativa a $5 \%$ de probabilidade pelo teste $t$ de Student.

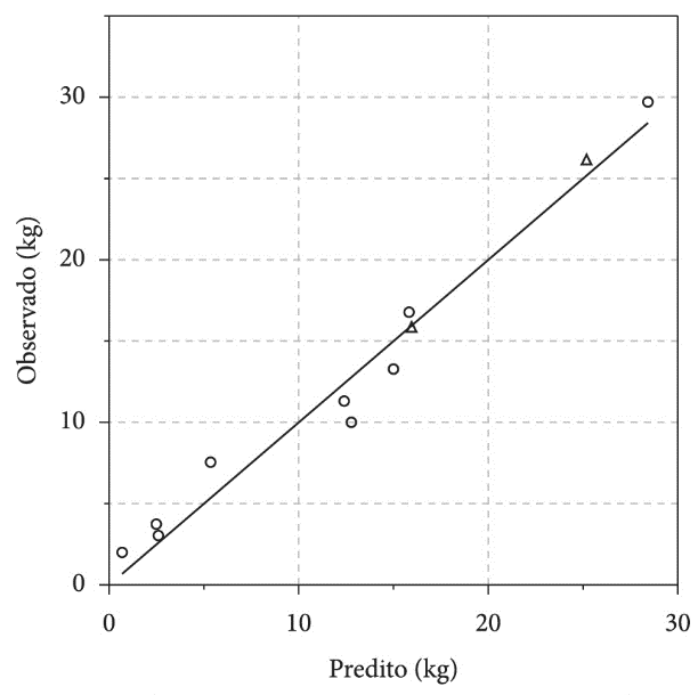

○ ○ ○ Fitomassa (campo)

$\Delta \Delta \Delta$ Fitomassa-teste (campo)

—_ Modelo ajustado_baseado $\mathrm{em} \mathrm{dh}^{2}$

Figura 2. Fitomassa predita versus observada no cultivo de pinhão manso em Taubaté, SP.

Figure 2. Biomass predicted and observed in Jatropha's crop in Taubaté, SP. 
O histograma dos resíduos (I) ilustra a distribuição dos resíduos em relação à fitomassa do pinhão manso mensurada em campo. A avaliação dessa dispersão não é conclusiva, fazendo com que a hipótese de normalidade dos resíduos não seja rejeitada por esse gráfico, devendo ser investigada com outros parâmetros.

Em (II), que apresenta justamente a aderência dos resíduos a essa distribuição normal dos dados, é possível avaliar essa questão, na qual não se encontrou evidência contra a normalidade dos resíduos, uma vez que os

Tabela 4. Anova da Equação 1, para estimativa da fitomassa do pinhão manso.

Table 4. Anova of Equation (1) to estimate the biomass of Jatropha.

\begin{tabular}{cccccc}
\hline \multicolumn{7}{c}{ Anova } \\
\hline Fatores & G.L. & SQ & $\mathbf{Q M}$ & Estat. $\boldsymbol{F}$ & p-valor \\
$\mathrm{h}^{2} \mathrm{~d}$ & 1 & 1630,5 & $1.630,5$ & 574,1 & $\mathrm{NS}$ \\
\hline \multicolumn{6}{c}{ Resíduos } \\
\hline Mínimo & $\mathbf{1}^{\circ} \mathbf{Q}$ & Média & Mediana & $3^{\circ} \mathbf{Q}$ & Máximo \\
\hline$-2,7$ & $-1,1$ & 1,0 & 0,2 & 1,4 & 2,2 \\
\hline
\end{tabular}

(I) Histograma dos resíduos

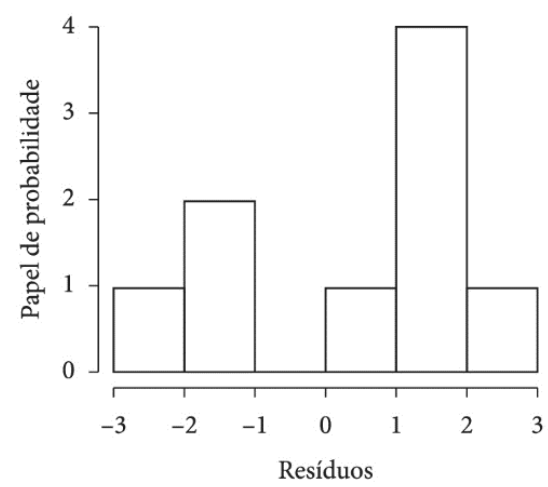

(III) Resíduos x Valores ajustados

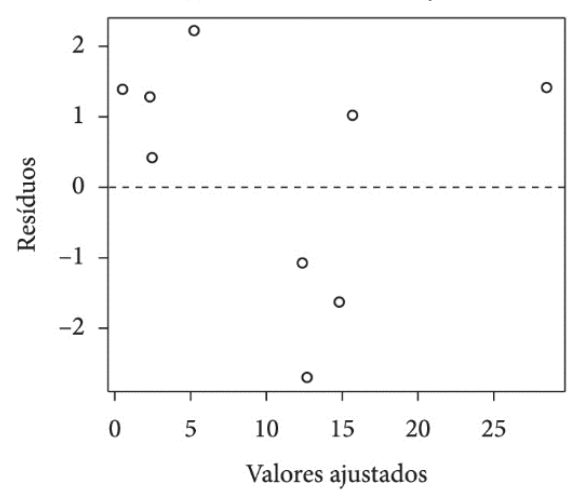

pontos estão todos em torno de uma linha reta. Além disso, como o p-valor é alto (aproximadamente 0,15 ), não foi rejeitada a hipótese de normalidade dos resíduos.

Não se detectou nenhum tipo de tendência na dispersão (III), apontando que a distribuição dos resíduos foi aleatória. Porém esperava-se que essa distribuição tendesse a zero e ressaltasse o bom ajuste da equação. Diante do critério de homoscedasticidade das variâncias, que mede a dispersão dos resíduos perante o modelo regredido, se observou que essa foi de aproximadamente $10 \%$ em relação à medida de campo.

Por meio de (IV) foi verificada a independência dos resíduos frente ao procedimento metodológico, no qual, se os pontos estivessem distribuídos segundo um padrão haveria indicativos de dependência nos resíduos em relação à massa medida, ou seja, teríamos indícios de que alguma variável "extra" influenciou nos resultados do experimento, violando uma das premissas da Anova.

(II) Papel de probabilidade

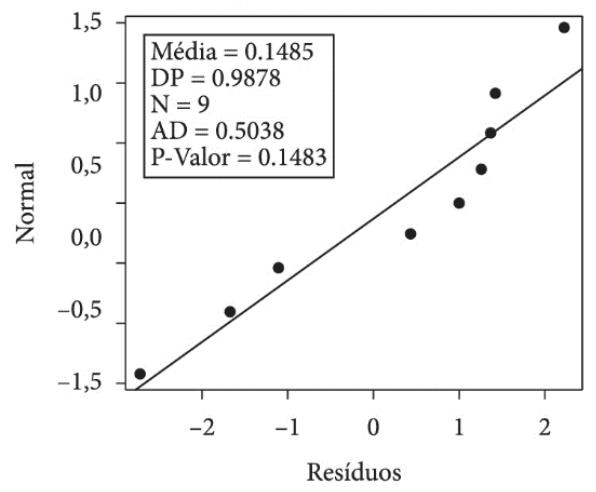

(IV) Resíduos x Ordem de coleta

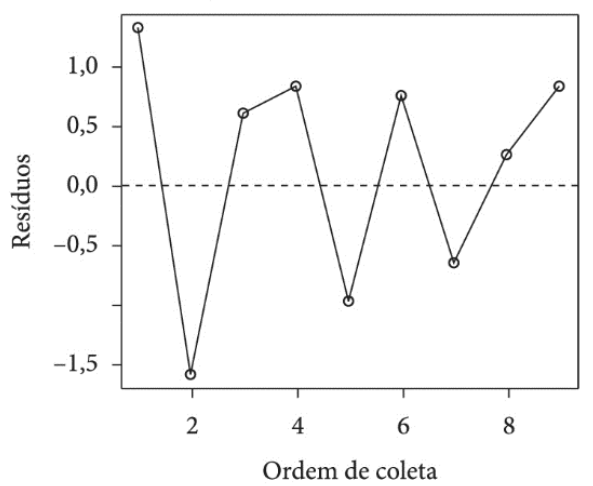

Figura 3. Análise de resíduos observados a partir dos dados gerados por meio da Equação 1. Figure 3. Analysis of residues observed from the data produced using Equation 1. 
Assim, a utilização da Equação 1 não apresentou erros estatísticos significativos, o que indica a possibilidade de seu uso na estimativa da fitomassa da cultura do pinhão manso por meio de variáveis de fácil mensuração em campo, como a altura e o diâmetro de projeção da copa.

Os resultados obtidos com a Equação 1 com outras três equações utilizadas para a estimativa da fitomassa de árvores de pinhão manso já publicadas podem ser visualizados na Figura 4, com base nas medições de campo realizadas no experimento, em Taubaté, SP.

Baseado na Figura 4, para árvores com estatura menor do que $2 \mathrm{~m}$ e fitomassa próxima de $20 \mathrm{~kg}$, os modelos fornecem estimativas muito semelhantes.

No entanto, a partir desse limite as equações previamente publicadas tendem a subestimar significativamente os valores, atribuindo-se isso ao fato de as estimativas terem sido realizadas contemplando exemplares menores do que 3,2 $\mathrm{m}$ de altura.

Cabe ressaltar que o modelo aqui proposto (Equação 1) pode ser utilizado não somente para árvores com maior estatura, mas também para estimativa da fitomassa para árvores de pinhão manso em fase inicial de crescimento.

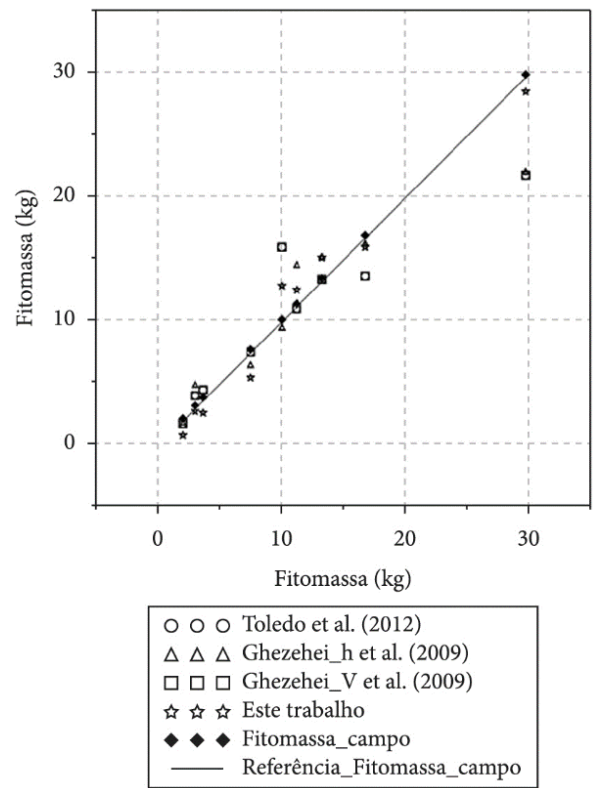

Figura 4. Comparativo entre modelos de estimativa da fitomassa do pinhão manso.

Figure 4. Comparative models to estimate the biomass of Jatropha.
O estudo realizado reforça a ideia de que a complexidade estrutural da planta oferece grandes entraves à busca por relações alométricas em cultivos de pinhão manso, muito influenciada por fatores regionais.

Em razão disso, cada qual em sua região, os autores proponentes das equações encontraram diferentes variáveis alométricas que mostraram correlação com a fitomassa e, embora altura e diâmetro respondam de maneira adequada nos diferentes sítios, o arranjo dessas variáveis pode ser alterado, de modo a atuar na precisão das medidas.

Ao assumir o espaçamento utilizado no experimento da APTA, o que corresponde a uma densidade de 833 árvores $\mathrm{ha}^{-1}$, e a massa seca determinada em laboratório, correspondente a $23 \%$ da massa úmida encontrada em campo, estimou-se que o estoque de fitomassa varia de $0,5 \mathrm{a} 6 \mathrm{Mg} \mathrm{ha}^{-1}$, enquanto o carbono armazenado, estimado com base na massa seca das plantas, atingiu como valor máximo aproximadamente $3 \mathrm{Mg} \mathrm{C} \mathrm{ha}^{-1}$. Com base nas equações previamente publicadas para essa mesma estimativa, o carbono estocado variaria entre 0,8 e 2,1 $\mathrm{Mg} \mathrm{C} \mathrm{ha}^{-1}$.

Comparativamente, o máximo de carbono estimado neste experimento na região do Vale do Paraíba Paulista foi inferior ao valor apresentado por Torres et al. (2011) $\left(4,2 \mathrm{Mg} \mathrm{C} \mathrm{ha}^{-1}\right)$ para o plantio de pinhão manso em Viçosa, MG, no espaçamento $3,5 \times 3 \mathrm{~m}$ (4 anos de idade). Por outro lado, o valor de carbono aqui estimado equivale ao encontrado por Reinhardt et al. (2007) para o pinhão manso na Índia, com 3,5 anos de idade, em cultivo de maior adensamento (espaçamento $3 \times 2 \mathrm{~m}$ ), com valor próximo de $3,2 \mathrm{Mg} \mathrm{C} \mathrm{ha}{ }^{-1}$.

É importante observar que a utilização de valores dos parâmetros mínimos e máximos na caracterização das árvores tende a subestimar ou superestimar os estoques de carbono, dependendo do caso. No entanto, essa estimativa preditiva se torna uma referência importante para os estudos de fitomassa e carbono no Vale do Paraíba e deve ter sua investigação continuada para que resultados mais precisos sejam alcançados, uma vez que a espécie tem atraído atenção mundial pelo relevante potencial como fonte de biocombustíveis.

A possibilidade de recuperar solos degradados usados para pastoreio com implantação de cultura para a produção de bioenergia faz com que a introdução comercial do pinhão manso no Vale do Paraíba seja bastante atrativa, pois como agente de sustentabilidade 
o pinhão manso pode oferecer aos pequenos e médios produtores rurais novas perspectivas sociais e econômicas, contribuindo para a melhoria das condições ambientais e fortalecendo, assim, o desenvolvimento regional.

\section{CONCLUSÕES}

No estudo realizado evidenciou-se a concepção de que a complexa forma estrutural das plantas de pinhão manso dificulta a busca por relações alométricas em áreas de cultivo, muito influenciadas por fatores locais.

Para o Vale do Paraíba do Sul, essas relações puderam ser ajustadas a um modelo preditivo linear simples e direto, sem erros estatísticos significativos.

A aplicação do modelo aqui proposto revelou que a massa seca da planta para árvores com 6 anos na área estudada varia de 0,5 a $6 \mathrm{Mg} \mathrm{ha}^{-1}$, enquanto que o carbono armazenado chega a atingir $3 \mathrm{Mg} \mathrm{C} \mathrm{ha-1}^{-}$.

\section{AGRADECIMENTOS}

Agradecemos o apoio financeiro do CNPq, Projeto 480990/2007-2 - Pinhão Manso (Jatropha curcas L.): Alternativa econômica, social e ambiental para a produção de biodiesel no Vale do Paraíba (SP) e ao engenheiro agrônomo José Luiz de Carvalho, do Instituto Florestal de São Paulo, pela cessão de equipamentos de campo e discussão dos resultados.

\section{STATUS DA SUBMISSÃO}

Recebido: 10 jan., 2013

Aceito: 21 mar., 2015

\section{AUTOR(ES) PARA CORRESPONDÊNCIA}

\section{Willian José Ferreira}

Centro de Ciência do Sistema Terrestre - CCST, Instituto Nacional de Pesquisas Espaciais - INPE, CEP 12319-580, São José dos Campos, SP, Brasil e-mail: willian.ferreira@inpe.br

\section{APOIO FINANCEIRO}

CNPq, Projeto 480990/2007-2.

\section{REFERÊNCIAS}

Brasil. Ministério de Ciência, Tecnologia e Inovação. Status dos projetos no âmbito do Mecanismo de Desenvolvimento Limpo (MDL) no Brasil e no mundo: $1^{\circ}$ período de compromisso do Protocolo de Quioto (2008-2012) [online]. 2014 [acesso em 2014 nov. 10]. Disponível em: http:// www.mct.gov.br/upd_blob/0232/232099.pdf

Castro AG, Carvalho CM, Cândido DM. Avaliação da germinação em viveiro da espécie Jatropha Curcas L. (pinhão manso) de distintas procedências. Taubaté/SP: Instituto Florestal de Taubaté; 2006.

Clough BF, Scott K. Allometric relationships for estimating above-ground biomass in six mangrove species. Forest Ecology and Management 1989; 27(2): 117-127. http:// dx.doi.org/10.1016/0378-1127(89)90034-0.

Cole TG, Ewel JJ. Allometric equations for four valuable tropical tree species. Forest Ecology and Management 2006; 229(1-3): 351-360. http://dx.doi.org/10.1016/j. foreco.2006.04.017.

Devide ACP, Castro CM, Rodrigues C Jr. Efeito de culturas de cobertura e da poda na produtividade do pinhão manso. In: Anais do IV Congresso Brasileiro de Mamona e I Simpósio Internacional de Oleaginosas Energéticas: Inclusão social e energia; 2010; João Pessoa. Campina Grande: Embrapa Algodão; 2010. p. 1326-1332.

Dewar RC, Cannell MGR. Carbon sequestration in the trees, products and soils of forest plantations: an analysis using UK examples. Tree Physiology 1992; 11(1): 49-71. http:// dx.doi.org/10.1093/treephys/11.1.49. PMid:14969967.

Dias LAS, Leme LP, Laviola BG, Pallin A, Pereira OL, Dias DCFS. Cultivo de pinhão manso (Jatropha curcas L.) para produção de óleo combustível. Viçosa: UFV; 2007. 40 p.

Escola de Administração de Empresas da Fundação Getulio Vargas - EAESP. Financiamentos públicos e mudança do clima: análise das estratégias e práticas de bancos públicos e fundos constitucionais brasileiros na gestão da mudança do clima [online]. São Paulo: Centro de Estudos em Sustentabilidade; 2012 [acesso em 2014 nov. 09]. 67 p. Disponível em: http://www.mma.gov.br/estruturas/251/_ publicacao/251_publicacao10062011045049.pdf

Fisch G. Distribuição da precipitação em Taubaté, Vale do Paraíba (SP). Revista Biociências 1999; 5(2): 7-11.

Ghezehei SB, Annandalea JG, Everson CS. Shoot allometry of Jatropha curcas. Southern Forests: a Journal of Forest Science 2009; 71(4): 279-286. http://dx.doi.org/10.2989/ SF.2009.71.4.5.1032.

Helou ARHA, Helou EA Fo, Otani N, Selig PM. Indicadores de sustentabilidade na gestão ambiental considerando um projeto de código florestal. Sustainable Business International Journal 2012; (22): 1-23.

Juhász ACP, Morais DLB, Soares BO, Pimenta SR, Rabello $\mathrm{H}$, Resende MDV. Parâmetros genéticos e ganho de seleção 
para populações de pinhão manso (Jatropha curcas). Pesquisa Florestal Brasileira 2010; 30(61): 25-35. http:// dx.doi.org/10.4336/2010.pfb.30.61.25.

Laviola BG, Bhering LL, Albrecht JC, Marques SS, Rosado-Laviola TB. Caracterização morfo-agronômica do banco de germoplasma de pinhão-manso. In: Anais do Congresso Brasileiro de Plantas, Oleaginosas, Óleos, Gorduras e Biodiesel; 2009; Montes Claros. Lavras/MG: UFLA; 2009. p. 1-2.

Leite HG, Andrade VCL. Importância das variáveis altura dominante e altura total em equações hipsométricas e volumétricas. Revista Árvore 2003; 27(3): 301-310. http:// dx.doi.org/10.1590/S0100-67622003000300005.

Medeiros R. Desafios à gestão sustentável da biodiversidade no Brasil. Floresta e Ambiente [online]. 2006 [acesso em 2014 nov. 12]; 13(2): 1-10. Disponível em: http://www. floram.org/files/v13n2/v13n2a1.pdf

Miranda DLC, Melo ACG, Sanquetta CR. Equações alométricas para estimativa de biomassa e carbono em árvores de reflorestamentos de restauração. Revista Árvore 2011; 35(3): 679-689. http://dx.doi.org/10.1590/S010067622011000400012 .

Mohan Kumar B, Jacob George SJ, Jamaludheen V, Suresh TK. Comparison of biomass production, tree allometry and nutrient use efficiency of multipurpose trees grown in woodlot and silvopastoral experiments in Kerala, India. Forest Ecology and Management 1998; 112(1-2): 145-163. http://dx.doi.org/10.1016/S0378-1127(98)00325-9.

Reinhardt G, Gärdner S, Rettenmaier N, Münch J, Von Falkenstein E. Screening life cycle assessment of Jatropha Biodiesel [online]. Heildelberg, DE: Institute for Energy and Environmental Research Heidelberg - IFEU; 2007 [acesso em 2014 nov. 12] 13 p. Disponível em: http://www. ifeu.de/landwirtschaft/pdf/jatropha_report_111207.pdf
Rolim SG, Couto HTZ, Jesus RM, França JT. Modelos volumétricos para a Floresta Nacional do Tapirapé-Aquirí, Serra dos Carajás (PA). Acta Amazonica 2006; 36(1): 107 114. http://dx.doi.org/10.1590/S0044-59672006000100013.

Silva DA, Marchiori F, Andrade C. Comportamento da semente de pinhão-manso (Jatropha curcas L.) sob diferentes temperaturas visando à utilização energética. Floresta e Ambiente 2012; 19(4): 414-421. http://dx.doi. org/10.4322/floram.2012.046.

Soares CPB, Paula F No, Souza AL. Dendrometria e inventário florestal. Viçosa/MG: Editora UFV; 2007.276 p.

Toledo DP, Jacovine LAG, Torres CMME, Soares SPB. Equação de biomassa e estoque de carbono do pinhão manso, no município de Viçosa, MG. Ciência Rural 2012; 42(11): 1998-2004. http://dx.doi.org/10.1590/S010384782012005000086 .

Torres CMME, Jacovine LAG, Toledo DP, Soares CPB, Ribeiro SC, Martins MC. Estoque de biomassa e de carbono de Jatropha curcas L. Cerne 2011; 17(3): 353-359. http:// dx.doi.org/10.1590/S0104-77602011000300009.

UN-REDD Programme. Un-Redd Programe: The United Nations Collaborative Programme on Reducing emissions from deforestation and forest degradation in developing countries. About un-redd [online]. 2012 [acesso em 2014 nov. 10]. Disponível em: http://www.un-redd.org/Home/ tabid/565/Default.aspx

Urbanska KM, Webb NR, Edwards PJ. Restoration ecology and sustainable development. Cambridge: Cambridge University Press; 2000. 397 p.

Watzlawick LF, Kircher FF, Sanquetta CR, Schumacher MV. Fixação de carbono em floresta ombrófila mista em diferentes estágios de regeneração. In: Sanquetta CR, Watzlawick LF, Balbinot R, Ziliotto MAB, Gomes FS, editores. As florestas e o carbono. Curitiba/PR: Imprensa Universitária da UFPR; 2002. 153-173. 